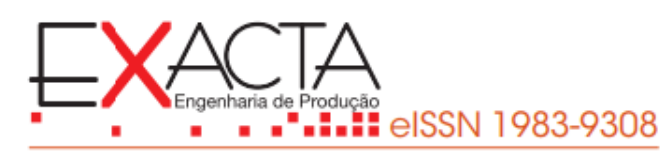

\title{
FATORES-CRÍTICOS DE SUCESSO EM PROJETOS DA INDÚSTRIA 4.0
}

\section{SUCCESS CRITICAL FACTORS IN INDUSTRY 4.0 PROJECTS}

Recebido em: 26 out. 2020

Aprovado em: 14 dez. 2020

Versão do autor aceita publicada online: 14 dez. 2020

Publicado online: 01 jul. 2021

Como citar esse artigo - American Psychological Association (APA):

Teixeira, S. C. A., Pedron, C. D., \& Silva, L. F. (2022, out./dez.). Fatores-críticos de sucesso em projetos da indústria 4.0. Exacta. 20(4), 884-899.

https://doi.org/10.5585/exactaep.2022.18581.

Submeta seu artigo para este periódico $\beta$

Dados Crossmark 


\title{
FATORES-CRÍTICOS DE SUCESSO EM PROJETOS DA INDÚSTRIA
} 4.0

\author{
SUCCESS CRITICAL FACTORS IN INDUSTRY 4.0 PROJECTS
}

\section{iD Silvio Cesar Alves Teixeira ${ }^{1}$ \\ (iD) Cristiane Drebes Pedron ${ }^{2}$ \\ iD \\ Luciano Ferreira da Silva ${ }^{3}$}

\author{
${ }^{1}$ Mestre em Administração \\ Universidade Nove de Julho (UNINOVE). \\ São Paulo, SP - Brasil. \\ silvio.1803@hotmail.com \\ 2 Doutora em Gestão \\ Universidade Nove de Julho (UNINOVE). \\ São Paulo, SP - Brasil. \\ cdpedron@gmail.com \\ 3 Doutor em Administração \\ Universidade Nove de Julho (UNINOVE). \\ São Paulo, SP - Brasil. \\ prof.Ifs7725@gmail.com
}

Recebido em: 26 out. 2020

Aprovado em: 14 dez. 2020
Resumo: O objetivo deste estudo foi identificar os Fatores Críticos de Sucesso (FCS) em projetos da Indústria 4.0, para apoiar a área de gestão de projetos no mapeamento e desenvolvimento de atividades durante as fases de iniciação, planejamento, execução e encerramento em projetos voltados para Indústria 4.0. O estudo adotou como estratégia de pesquisa uma Revisão Sistemática da Literatura (RSL) cuja análise foi constituída por 56 artigos, devidamente categorizados, o que permitiu construir matrizes comparativas. Como principais resultados foram mapeados 53 Fatores Críticos de Sucesso (FCS), agrupados em 10 categorias conforme características de cada fator. Como contribuições teóricas e sociais, o estudo disponibiliza uma relação de FCS e sua influência em projetos da Indústria 4.0, cujo a aplicabilidade pode auxiliar a área de gestão de projetos durante todas as fases do projeto.

Palavras-chave: Gerenciamento de Projetos. Fatores Críticos de Sucesso. FCS. RSL. Indústria 4.0.

Abstract: The aim of this study was to identify the Critical Success Factors (CSF) in Industry 4.0 projects, to support the project management area in mapping and developing activities during the initiation, planning, execution and closing phases in projects focused on Industry. 4.0 The study adopted as a research strategy a Systematic Literature Review (RSL) whose analysis consisted of 56 articles, properly categorized, which allowed the construction of comparative matrices. As main results, 53 Critical Success Factors (CSF) were mapped, grouped into 10 categories according to the characteristics of each factor. As theoretical and social contributions, this study provides a list of FCS and its influence on Industry 4.0 projects, whose applicability can help the project management area during all phases of the project.

Keywords: Project Management. Critical Success Factor. FCS. RSL. Industry 4.0. 


\section{Introdução}

Com o advento da Indústria 4.0, a comunidade de gestão de projetos inicia uma série de debates sobre de que forma as novas tecnologias deverão afetar o gerenciamento de projetos. Marnewick e Marnewick (2019) argumentam que a Indústria 4.0 afetará o processo de gerenciamento de projetos, o ambiente de trabalho no qual os projetos serão executados, assim como, os membros do time de projetos que terão que se adaptar aos novos processos e novas tecnologias, sejam elas, físicas ou virtuais.

Essa mesma Indústria 4.0 tem elevado o volume de iniciativas e projetos, sendo que as empresas demonstram interesse em mapear os fatores fundamentais para evoluir nesse contexto. Matt e Benlian (2015) denominam esta transformação como estratégia digital, a qual tem como finalidade avançar em inovação. Cakmakci (2019) menciona que as mudanças tecnológicas representam uma nova organização cujo principal objetivo é atender às necessidades dos clientes. Portanto, mapear os fatores críticos em projetos considerando a realidade e características da Indústria 4.0 é importante para que se obtenha projetos com resultados positivos.

Radujković e Sjekavica (2017) estudaram a gestão de projetos para identificar os fatores de sucesso e demonstram que as competências também são consideradas Fatores Críticos de Sucesso (FCS). Os autores concluíram que mapear os FCS possibilita uma visão clara das necessidades dos projetos e o alcance dos resultados esperados.

O estudo realizado por Osei-Kyei e Chan (2015) apresenta uma revisão da literatura sobre FCS em projetos entre 1990 a 2013, com foco em parceiras público-privadas. Este trabalho demonstra que a relevância dos FCS em projetos e apresenta uma linha crescente de publicações sobre esta temática. Os autores concluem nesse estudo que apenas planejar, organizar, monitorar e controlar projetos não são suficientes e recomendam um aprofundamento dos FCS em gestão de projetos.

Considerando esta situação, o presente estudo procura responder a seguinte questão de pesquisa: Quais são os fatores críticos de sucesso dos projetos da Indústria 4.0? Para tanto, foi realizada uma Revisão Sistemática da Literatura (RSL) com o objetivo de mapear os FCS em projetos da Indústria 4.0. A contribuição deste estudo se baseia na apresentação de fatores que apoiam a área de gestão de projetos no mapeamento e desenvolvimento de atividades durante as fases de iniciação, planejamento, execução e finalização em projetos da Indústria 4.0. Slusarczykk (2018) argumenta que a Indústria 4.0 é uma realidade econômica moderna e os FCS são pontos estratégicos para as organizações, o que também justifica a importância deste estudo.

Este estudo apresenta a metodologia utilizada, seguido da apresentação e análise dos resultados, e das considerações finais. 


\section{Metodologia}

O estudo foi efetuado a partir de uma RSL buscando entender os FCS presentes em projetos de tecnologias relacionados à Indústria 4.0, aqui denominados tecnologias 4.0. Segundo Pollock e Berge (2018), uma RSL visa reunir evidências para responder a uma pergunta de pesquisa pré-definida e envolve a identificação de toda a pesquisa relevante, a avaliação crítica e a síntese dos achados. Os autores também relatam a importância de um protocolo como parte essencial do processo de revisão porque deve incluir informações suficientes para habilitar a replicação independente dos métodos.

A busca por documentos para análise foi efetuada na base de dados Scopus por intermédio dos seguintes termos: (("Critical Success Factor*" OR "Success Factor*") AND ("industr* tranformation*" OR "industr* Innovation*" OR "ind 4.*" OR "industr* 4.*" OR "smart indust*" OR "digital industr*")). A busca resultou em 1733 artigos. Da mesma forma, foi efetuada uma busca na base de dados do Web of Science a qual resultou em 29 documentos. A princípio não foi aplicado nenhum filtro e a fase inicial teve como foco comparar os documentos das duas bases. Como resultado observou-se que todos os arquivos existentes no Web of Science estavam presentes no Scopus, sendo assim foi considerado apenas a base Scopus como referência.

Em uma RSL, os critérios de seleção são determinantes para um resultado efetivo e devem ser alinhados à forma de avaliação para construção de uma base sólida de artigos, permitindo assim o desenvolvimento de uma discussão concisa (Russo \& Camanho, 2015). Após considerar somente a base Scopus, foram aplicados os critérios para selecionar quais documentos realmente abordavam os temas relacionados à FCS em projetos de tecnologias 4.0 que de fato contribuíssem para o desenvolvimento da discussão para apresentar quais as categorias que possuem maior concentração de FCS em projetos da Indústria 4.0.

Os seguintes critérios de inclusão foram aplicados: (1) artigos que explicitamente contêm FCS no contexto estudado; (2) artigos cujo conteúdo aborda FCS para resultados ou estudos de casos; e (3) artigos com volume significativo de citações. Os seguintes critérios de exclusão foram aplicados: (1) livros; (2) idiomas diferentes de inglês e português; (3) artigos anteriores à 2014; e (4) artigos com nenhuma citação, com exceção dos publicados em 2019. Os documentos que não atenderam critérios de inclusão ou exclusão foram excluídos porque os mesmos foram caracterizados como não tendo relação com o tema.

Após aplicar estes critérios, foram identificados 372 documentos. Foram analisados todos os títulos e resumos, tendo resultado em 56 documentos conforme Figura 1. 
Figura 1

\section{Processo Para Seleção dos Documentos}

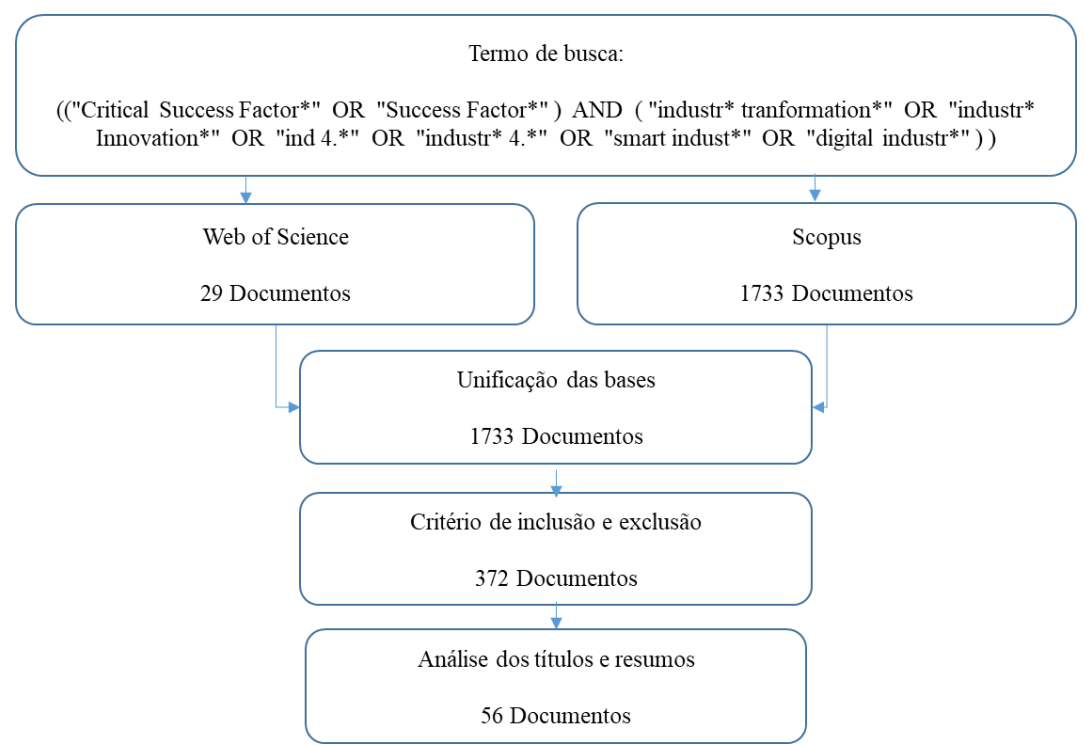

Fonte: Adaptado de Pollock e Berge (2018).

Os 56 artigos selecionados para análise mais aprofundada passaram por uma leitura crítica e categorização. O processo de categorização permitiu construir matrizes comparativas para explicar similitudes e diferenças no corpus de análise. Cabe explicitar que este não é um processo contínuo e recursivo de categorização, validação, comparação e agrupamento. Os resultados desta análise são apresentados na próxima seção.

\section{Os fatores críticos de sucesso dos projetos da Indústria 4.0}

O estudo mapeou 53 FCS presentes nos 56 artigos analisados na RSL. De acordo com a característica de cada FCS, foi possível agrupá-los em 10 categorias conforme Tabela 1. A categorização dos FCS possibilitou entender onde estão concentrados o maior volume de FCS e desta forma, identificar quais são as categorias de maior influência em projetos da Indústria 4.0. 
Tabela 1

FCS Mapeados e Categoria

\begin{tabular}{|c|c|c|c|c|c|c|c|c|c|c|c|}
\hline FCS & 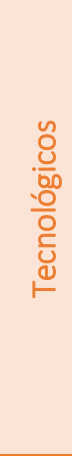 & 음 & 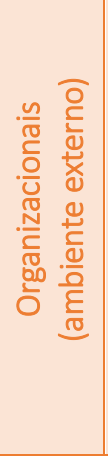 & 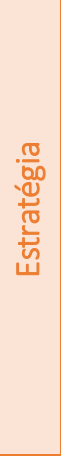 & 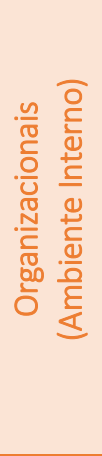 & 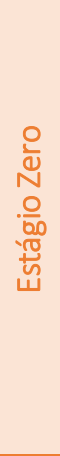 & 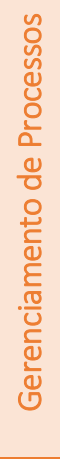 & $\begin{array}{l}\frac{n}{\sqrt{2}} \\
\frac{ \pm}{3} \\
\end{array}$ & 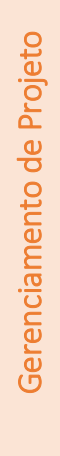 & 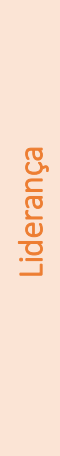 & $\frac{\overline{0}}{\frac{\pi}{4}} \frac{0}{\frac{0}{0}}$ \\
\hline Escolha da tecnologia & 28 & & & & & & & & & & 28 \\
\hline Performance dos sistemas & 20 & & & & & & & & & & 20 \\
\hline Treinamento e Capacitação & & 19 & & & & & & & & & 19 \\
\hline Integração dos Sistemas & 17 & & & & & 1 & & & & & 18 \\
\hline Segurança da Informação & 17 & & & & & & & & & & 17 \\
\hline Capital Financeiro & & & & & 16 & & & & & & 16 \\
\hline Arquitetura dos sistemas & 14 & & & & & & & & & & 14 \\
\hline Inovação & & & & 13 & & & & & & & 13 \\
\hline Cenário atual & & & & & & 12 & & & & & 12 \\
\hline Perfil da Organização & & & & & & & & 11 & & & 11 \\
\hline Fornecedores & & & 8 & & & & & & & & 8 \\
\hline Sustentabilidade & & & 8 & & & & & & & & 8 \\
\hline Metodologia & & & & & & & 8 & & & & 8 \\
\hline Disponibilidade dos sistemas & 8 & & & & & & & & & & 8 \\
\hline Alinhamento Estratégico & & & & 8 & & & & & & & 8 \\
\hline Suporte Técnico & 6 & & & & & & & & & & 6 \\
\hline Desenvolvimento dos sistemas & 6 & & & & & & & & & & 6 \\
\hline Governamentais & & & 6 & & & & & & & & 6 \\
\hline Gestão do conhecimento & & & 2 & & 3 & & & & & & 5 \\
\hline Economia Circular & & & 5 & & & & & & & & 5 \\
\hline Comprometimento da Liderança & & & & & & & & & & 5 & 5 \\
\hline Resultado & & & & 2 & & & & & 3 & & 5 \\
\hline Automação & 5 & & & & & & & & & & 5 \\
\hline Políticas & & & & & & & 5 & & & & 5 \\
\hline Intensidade Tecnológica & & & & & & 4 & & & & & 4 \\
\hline Engajamento & & 4 & & & & & & & & & 4 \\
\hline Escalabilidade & 4 & & & & & & & & & & 4 \\
\hline Escolha da equipe & & 4 & & & & & & & & & 4 \\
\hline Trabalho em Equipe & & 4 & & & & & & & & & 4 \\
\hline Gestão descentralizada & & & & & 3 & & 1 & & & & 4 \\
\hline Colaboração & & 4 & & & & & & & & & 4 \\
\hline Satisfação do Cliente & & & 3 & & & & & & & & 3 \\
\hline Comunicação & & & & & & & & & 3 & & 3 \\
\hline Empoderamento & & & & & 3 & & & & & & 3 \\
\hline Carga de Trabalho & & 3 & & & & & & & & & 3 \\
\hline Estrutura Formal & & & & & 3 & & & & & & 3 \\
\hline Sistemas de Colaboração & 2 & & & & & & & & & & 2 \\
\hline Gerenciamento do Projeto & & & & & & & & & 2 & & 2 \\
\hline
\end{tabular}

Exacta, 20(4), p. 884-899, out./dez. 2022 


\begin{tabular}{|c|c|c|c|c|c|c|c|c|c|c|c|}
\hline FCS & 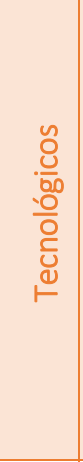 & $\frac{\stackrel{ }{\frac{0}{3}}}{\text { 뭄 }}$ & 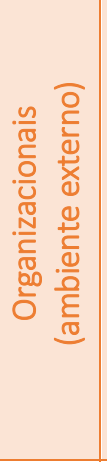 & 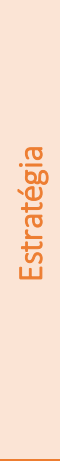 & 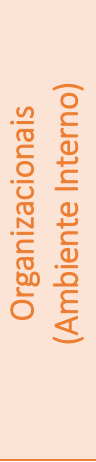 & 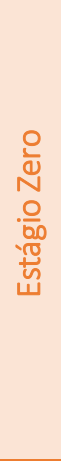 & 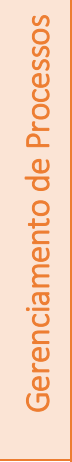 & $\begin{array}{l}\frac{n}{\pi} \\
\frac{2}{3} \\
\frac{D}{3}\end{array}$ & 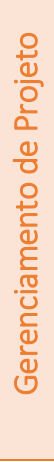 & 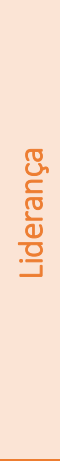 & 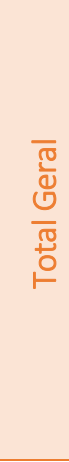 \\
\hline Qualidade & & & & 2 & & & & & & & 2 \\
\hline Realocação de Profissionais & & 2 & & & & & & & & & 2 \\
\hline Estrutura Informal & & & & & 2 & & & & & & 2 \\
\hline Ciclo de Vida do Produto & & & & 2 & & & & & & & 2 \\
\hline Região & & & & & & & & 2 & & & 2 \\
\hline Alianças & & & & & & & & & & 2 & 2 \\
\hline Produção distribuída & & & & 2 & & & & & & & 2 \\
\hline Gestão Centralizada & & & & & & & 1 & & & & 1 \\
\hline Monitoração dos Sistemas & 1 & & & & & & & & & & 1 \\
\hline Riscos & & & & & & & & & 1 & & 1 \\
\hline Reengenharia de Processos & & & & & & 1 & & & & & 1 \\
\hline Governança & & & & & & & 1 & & & & 1 \\
\hline Padronização & & & & & & & 1 & & & & 1 \\
\hline Influência da Liderança & & & & & & & & & & 1 & 1 \\
\hline Empreendedorismo & & & & 1 & & & & & & & 1 \\
\hline Total Geral & 128 & 40 & 32 & 30 & 30 & 18 & 17 & 13 & 9 & 8 & 325 \\
\hline
\end{tabular}

Fonte: Autor.

Os 53 FCS contribuem de forma positiva para o sucesso dos projetos presentes nos estudos desta RSL e demonstram a influência de cada fator conforme sua especificidade técnica ou de mercado de forma que reforça a necessidade de entender e mapear os FCS no início de qualquer projeto da Indústria 4.0.

A análise da categorização demonstra uma maior concentração de FCS tecnológicos, principalmente com relação aos aspectos como escolha da tecnologia, performance, integração, segurança e arquitetura, seguidos de outros fatores considerados menos relevantes e que de modo geral podemos entender que é natural a maior concentração de FCS nesta categoria pelo fato de ser de projetos de tecnologia desta nova era. Mesmo com menor volume de FCS, a categoria Estágio Zero também está relacionada à tecnologia, mas é considerado como ponto de partida e não necessariamente como um item tecnológico, tem relevância para entender qual o estágio da tecnologia existente para entender quais são as necessidades e etapas para migrar para um estágio tecnológico mais avançado.

A segunda categoria com maior volume de FCS é relacionado ao fator humano que envolve a readequação dos profissionais para atuar com estas novas tecnologias e de forma integrada e colaborativa, além de pensar em como realocar as pessoas e reter conhecimento. De certa forma é 
possível relacionar que os FCS em Gestão de Processos, como metodologias de trabalho, novas políticas, padronizações, governança, além da escolha entre uma gestão centralizada ou descentralizada, podem também afetar o fator humano diretamente pelo fato das mudanças e indiretamente pela questão de insegurança. O fator humano também está relacionado com fatores culturais pelo relacionados diretamente ao perfil da empresa sendo ela inovadora ou com foco em eficiência.

A estratégia como FCS destacada pela inovação pode estar diretamente associada à capacidade financeira destacada em FCS organizacionais (ambiente interno), uma vez que, os estudos foram de empresas em casos empíricos em empresas de grande porte, assim como a necessidade de alinhamento estratégico para implantação de projetos da Indústria 4.0. Já os FCS relacionados à organização (ambiente externo) demonstram uma questão social e política pela sustentabilidade e fatores governamentais, além de uma preocupação com os fornecedores com relação a fornecimento da solução e manutenção ao longo do tempo.

A liderança está relacionada como um ponto de suporte e apoio durante os projetos do que propriamente um movimento contínuo participativo, assim como de certa forma o gerenciamento do projeto também exerce esse papel, mas como um foco em manter a comunicação bem estabelecida, controlar os riscos para atingir o resultado de implantação esperado.

A diferença na quantidade de FCS apresentadas na RSL, conforme demonstra a Tabela 2, é devido à característica do projeto estudado, aprofundamento do autor perante o estudo e em alguns casos o próprio objetivo, ou seja, quando mais profundo o estudo e mais próximo da tecnologia e da organização, mais fatores devem ser considerados. Em casos cujo objetivo é sustentabilidade ambiental, o próprio resultado é o FCS.

Tabela 2

Autores e FCS Mapeados em Cada Documento

\begin{tabular}{|c|c|c|}
\hline Categoria & FCS & Autores \\
\hline Tecnológicos & $\begin{array}{l}\text { Escolha da Tecnologia } \\
\text { Performance dos Sistemas } \\
\text { Integração dos Sistemas } \\
\text { Segurança da Informação } \\
\text { Arquitetura dos Sistemas } \\
\text { Disponibilidade dos Sistemas } \\
\text { Suporte Técnico } \\
\text { Desenvolvimento dos Sistemas } \\
\text { Automação } \\
\text { Escalabilidade } \\
\text { Sistemas de Colaboração } \\
\text { Monitoração dos Sistemas }\end{array}$ & $\begin{array}{l}\text { Abdel-Basset et al. (2018); Ardito et al. (2015); } \\
\text { Baccarelli et al. (2017); Bányai et al. (2019); } \\
\text { Bhagawati et al. (2019); Dalenogare et al. (2018); } \\
\text { Fengque et al. (2017); Frank et al. (2019); } \\
\text { Ghobakhloo (2019); Haseeb et al. (2019); Ji et al. } \\
\text { (2016); Kamble et al. (2018); Kamble, Gunasekaran e } \\
\text { Sharma (2018); Ključnikov et al. (2019); Li et al. } \\
\text { (2017); Liu et al. (2019); Lu et al. (2017); Lucianetti et } \\
\text { al. (2018); Luthra e Mangla (2018); Manavalan e } \\
\text { Jayakrishna (2019); Masood e Egger (2019); Moeuf } \\
\text { et al. (2018); Moktadir et al. (2018); Nagy et al. } \\
\text { (2018); Piccarozzi et al. (2018); Rajput e Singh (2019); } \\
\text { Rossini et al. (2019); Sevinç et al. (2018); Shinohara } \\
\text { et al. (2017); Singh, Misra e Chan (2019); Slusarczyk } \\
\text { et al. (2019); Tao e Zhang (2017); Theorin et al } \\
\text { (2017); Tsai (2018); Tsai e Lu (2018); Türkeș et al. }\end{array}$ \\
\hline
\end{tabular}




\begin{tabular}{|c|c|c|}
\hline Categoria & FCS & Autores \\
\hline & & $\begin{array}{l}\text { (2019); Varela et al. (2019); Xu et al. (2018); Zhang et } \\
\text { al. (2016). }\end{array}$ \\
\hline Equipe & $\begin{array}{l}\text { Treinamento e Capacitação } \\
\text { Escolha da Equipe } \\
\text { Colaboração } \\
\text { Trabalho em Equipe } \\
\text { Engajamento } \\
\text { Carga de Trabalho } \\
\text { Realocação de Profissionais }\end{array}$ & $\begin{array}{l}\text { Ardito et al. (2015); Bogers et al. (2017); Cezarino et } \\
\text { al. (2019); de Sousa Jabbour et al. (2018); } \\
\text { Ghobakhloo (2019); Huang et al. (2019); Kamble et } \\
\text { al. (2018); Kamble, Gunasekaran e Sharma (2018); } \\
\text { Kirchberger e Pohl (2016); Luthra e Mangla (2018); } \\
\text { Masood e Egger (2019); Moktadir et al. (2018); Nagy } \\
\text { et al. (2018); Piccarozzi et al. (2018); Sevinç et al. } \\
\text { (2018); Shinohara et al. (2017); Singh, Misra e Chan } \\
\text { (2019); Stachová et al. (2019); Türkeș et al. (2019). }\end{array}$ \\
\hline $\begin{array}{l}\text { Organizacionais } \\
\text { (ambiente externo) }\end{array}$ & $\begin{array}{l}\text { Sustentabilidade } \\
\text { Fornecedores } \\
\text { Governamentais } \\
\text { Economia Circular } \\
\text { Satisfação do Cliente } \\
\text { Gestão do Conhecimento }\end{array}$ & $\begin{array}{l}\text { Al-Shareem, Yusof e Kamal (2015); Ardito et al. } \\
\text { (2015); Bhagawati et al. (2019); Bogers et al. (2017); } \\
\text { Bonilla et al. (2018); Cezarino et al. (2019); Kamble et } \\
\text { al. (2018); Kamble, Gunasekaran e Sharma (2018); } \\
\text { Kirchberger e Pohl (2016); Lucianetti et al. (2018); } \\
\text { Luthra e Mangla (2018); Moktadir et al. (2018); } \\
\text { Piccarozzi et al. (2018); Rajput e Singh (2019); } \\
\text { Scuotto et al. (2017); Sevinç et al. (2018); Shinohara } \\
\text { et al. (2017); Tsai e Lu (2018); Varela et al. (2019). }\end{array}$ \\
\hline Estratégia & $\begin{array}{l}\text { Inovação } \\
\text { Alinhamento Estratégico } \\
\text { Ciclo de Vida do Produto } \\
\text { Produção Distribuída } \\
\text { Qualidade } \\
\text { Resultado } \\
\text { Empreendedorismo }\end{array}$ & $\begin{array}{l}\text { Ardito et al. (2015); Bhagawati et al. (2019); Bogers } \\
\text { et al. (2017); Cezarino et al. (2019); de Sousa Jabbour } \\
\text { et al (2018); Durana et al. (2019); Fettermann et al. } \\
\text { (2018); Ghobakhloo (2018); Ghobakhloo (2019); } \\
\text { Huang et al. (2019); Kirchberger e Pohl (2016); } \\
\text { Lucianetti et al. (2018); Moktadir et al. (2018); Nagy } \\
\text { et al. (2018); Nicoletti e Padovano (2019); Piccarozzi } \\
\text { et al. (2018); Rauch et al. (2017); Sevinç et al. (2018); } \\
\text { Shinohara et al. (2017); Singh, Misra e Chan (2019); } \\
\text { Tsai (2018); Türkeș et al. (2019). }\end{array}$ \\
\hline $\begin{array}{l}\text { Organizacionais } \\
\text { (Ambiente Interno) }\end{array}$ & $\begin{array}{l}\text { Capital Financeiro } \\
\text { Gestão Descentralizada } \\
\text { Estrutura Formal } \\
\text { Empoderamento } \\
\text { Gestão do Conhecimento } \\
\text { Estrutura Informal }\end{array}$ & $\begin{array}{l}\text { Ardito et al. (2015); Bhagawati et al. (2019); Bogers } \\
\text { et al. (2017); Cezarino et al. (2019); de Sousa Jabbour } \\
\text { et al. (2018); Ghobakhloo (2019); Huang et al. (2019); } \\
\text { Kamble, Gunasekaran e Sharma (2018); Kirchberger } \\
\text { e Pohl (2016); Lucianetti et al. (2018); Luthra e } \\
\text { Mangla (2018); Manavalan e Jayakrishna (2019); } \\
\text { Moktadir et al. (2018); Piccarozzi et al. (2018); Rossini } \\
\text { et al. (2019); Scuotto et al. (2017); Sevinç et al. } \\
\text { (2018); Shinohara et al. (2017); Singh, Misra e Chan } \\
\text { (2019); Slusarczyk (2018). }\end{array}$ \\
\hline Estágio Zero & $\begin{array}{l}\text { Cenário Atual } \\
\text { Intensidade Tecnológica } \\
\text { Integração dos Sistemas } \\
\text { Reengenharia de Processos }\end{array}$ & $\begin{array}{l}\text { Al-Shareem, Yusof e Kamal (2015); Bogers et al. } \\
\text { (2017); de Sousa Jabbour et al. (2018); Ghobakhloo } \\
\text { (2019); Kamble, Gunasekaran e Sharma (2018); } \\
\text { Kirchberger e Pohl (2016); Masood e Egger (2019); } \\
\text { Moktadir et al. (2018); Nagy et al. (2018); Rossini et } \\
\text { al. (2019); Singh, Misra e Chan (2019); Slusarczyk } \\
\text { (2018); Xu et al. (2018). }\end{array}$ \\
\hline $\begin{array}{l}\text { Gerenciamento de } \\
\text { Processos }\end{array}$ & $\begin{array}{l}\text { Metodologia } \\
\text { Políticas } \\
\text { Padronização } \\
\text { Gestão Descentralizada } \\
\text { Governança } \\
\text { Gestão Centralizada }\end{array}$ & $\begin{array}{l}\text { Bogers et al. (2017); Buer et al. (2018); Cezarino et al. } \\
\text { (2019); Kamble et al. (2019); Kamble, Gunasekaran e } \\
\text { Sharma (2018); Kirchberger e Pohl (2016); Lucianetti } \\
\text { et al. (2018); Luthra e Mangla (2018); Piccarozzi et al. } \\
\text { (2018); Rajput e Singh (2019); Rossini et al. (2019); } \\
\text { Shinohara et al. (2017); Singh, Misra e Chan (2019); } \\
\text { Tsai e Lu (2018); Xu et al. (2018). }\end{array}$ \\
\hline
\end{tabular}




\begin{tabular}{|c|c|c|}
\hline Categoria & FCS & Autores \\
\hline Culturais & $\begin{array}{l}\text { Perfil da Organização } \\
\text { Região }\end{array}$ & $\begin{array}{l}\text { Cezarino et al. (2019); de Sousa Jabbour et al. (2018); } \\
\text { Kamble, Gunasekaran e Sharma (2018); Kirchberger } \\
\text { e Pohl (2016); Luthra e Mangla (2018) } \\
\text { Masood e Egger (2019); Piccarozzi et al. (2018); } \\
\text { Rossini et al. (2019); Sevinç et al. (2018); Singh, Misra } \\
\text { e Chan (2019); Türkeș et al. (2019). }\end{array}$ \\
\hline $\begin{array}{l}\text { Gerenciamento de } \\
\text { Projeto }\end{array}$ & $\begin{array}{l}\text { Comunicação } \\
\text { Resultado } \\
\text { Gerenciamento do Projeto } \\
\text { Riscos }\end{array}$ & $\begin{array}{l}\text { de Sousa Jabbour et al. (2018); Kirchberger e Pohl } \\
\text { (2016); Sevinç et al. (2018); Shinohara et al. (2017); } \\
\text { Singh, Misra e Chan (2019); Slusarczyk et al. (2019). }\end{array}$ \\
\hline Liderança & $\begin{array}{l}\text { Comprometimento da Liderança } \\
\text { Alianças } \\
\text { Influência da Liderança }\end{array}$ & $\begin{array}{l}\text { de Sousa Jabbour et al. (2018); Kirchberger e Pohl } \\
\text { (2016); Sevinç et al. (2018); Shinohara et al. (2017); } \\
\text { Singh, Misra e Chan (2019). }\end{array}$ \\
\hline
\end{tabular}

Fonte: Autor.

Ao analisar a Tabela 2 pode ser evidenciado o agrupamento dos FCSs em suas 10 categorias e os respectivos pesquisadores. A seguir são tecidas discussões sobre os agrupamentos propostos.

\section{Discussão}

Cabe destacar que as empresas possuem realidades distintas e a organização das informações aqui apresentadas contribuem para uma melhor compreensão dos principais elementos de cada ponto crítico. Nesta linha de pensamento, destaca-se, por exemplo, a categoria "tecnológico" que elenca desde a escolha da tecnologia, a escalabilidade e o monitoramento do sistema, entre outros fatores intermediários.

Com o agrupamento e categorização dos FCS em projetos da Indústria 4.0, podemos observar que mesmo não sendo uma categoria com maior volume de FCS, o estágio zero é o ponto de partida para o entendimento no estado atual tecnológico da empresa para implantação de novas tecnologias. Em seguida é necessário avaliar os fatores tecnológicos e o fator humano, para não gerar conflitos e perda de conhecimento, ou ainda gerar barreiras durante as implantações.

Um terceiro bloco de categorias foi a estratégia, liderança e fatores organizacionais e culturais, que também devem ser analisadas para que os projetos tenham o apoio da alta gestão. Os impactos internos e externos a organização e o gerenciamento de projetos como FCS, tem um papel importante para atingir o resultado esperado. O mapeamento e a categorização dos FCS trazem uma melhor compreensão e análise de projetos da Indústria 4.0 seja no planejamento ou durante a implantação do projeto. 


\section{Considerações finais}

Este estudo se propôs a responder, por meio de uma RSL, a seguinte questão: "Quais são os fatores críticos de sucesso dos projetos da Indústria 4.0?". Ao final do estudo, considerando o corpus de 56 artigos, chegamos a proposta de 53 fatores, agrupados em 10 categorias.

A leitura em profundidade dos artigos permitiu o entendimento que parte significativa das empresas não estão prontas para implantar ou avançar na Indústria 4.0 por falta de conhecimento sobre os fatores críticos de sucesso, ou seja, das necessidades organizacionais para evoluir neste tipo de iniciativa. Por exemplo, fatores tecnológicos como escolha da tecnologia ou integração de sistemas é crítico em projetos da Indústria 4.0, assim como fatores relacionados a equipe de trabalho, tais como treinamento e engajamento, também são relevantes.

A contribuição do artigo para a academia refere-se ao mapeamento dos artigos encontrados sobre o tema. Dado que o tema ainda é exploratório, a consolidação dos FCS de forma sistematizada pode auxiliar outros pesquisadores interessados no assunto. Já como contribuição para os praticantes, considera-se que os FCS mapeados neste artigo auxiliam os gestores a melhor reconhecer a situação da empresa sobre a Indústria 4.0. Uma forma de preparar as empresas para esta nova realidade é conhecer os parâmetros e informações primordiais que auxiliam no planejamento, construção e avanço da Indústria 4.0 (De Carolis, Macchi, Negri, \& Terzi, 2017b). Este estudo traz uma gama de FCS que podem auxiliar em várias etapas quando se há o objetivo de implantar ou avançar na Indústria 4.0.

O estudo disponibiliza uma relação de FCS e sua influência em projetos da Indústria 4.0, cuja aplicabilidade pode auxiliar a área de gestão de projetos durante todas as fases do projeto. Como proposta de estudo futuro, recomendamos um estudo de campo para identificar o grau de impacto de cada categoria mapeada nesta pesquisa e a influência de cada FCS nas diferentes fases de um projeto.

\section{Referências}

Abdel-Basset, M., Manogaran, G., \& Mohamed, M. (2018). Internet of Things (IoT) and its impact on supply chain: A framework for building smart, secure and efficient systems. Future Generation Computer Systems, 86, 614-628. https://doi.org/10.1016/j.future.2018.04.051

Albers, A., Gladysz, B., Pinner, T., Butenko, V., \& Stürmlinger, T. (2016). Procedure for defining the system of objectives in the initial phase of an industry 4.0 project focusing on intelligent quality control systems. Procedia Cirp, 52, 262-267. https://doi.org/10.1016/j.procir.2016.07.067

Almada-Lobo, F. (2015). The Industry 4.0 revolution and the future of manufacturing execution systems (MES). Journal of Innovation Management, 3(4), 16-21.

https://doi.org/10.24840/2183-0606_003.004_0003 
Al-Shareem, K. M., Yusof, N. A., \& Kamal, E. M. (2015). External factors influencing the readiness for implementing public-private partnerships among public and private organizations in Yemen. Journal of Science e Technology Policy Management, 6(1), 56-75. https://doi.org/10.1108/JSTPM-07-2014-0030

Ardito, L., Messeni Petruzzelli, A., \& Albino, V. (2015). From technological inventions to new products: A systematic review and research agenda of the main enabling factors. European Management Review, 12(3), 113-147. https://doi.org/10.1111/emre.12047

Baccarelli, E., Naranjo, P. G. V., Scarpiniti, M., Shojafar, M., \& Abawajy, J. H. (2017). Fog of everything: Energy-efficient networked computing architectures, research challenges, and a case study. IEEE Access, 5, 9882-9910. https://doi.org/10.1109/ACCESS.2017.2702013

Bányai, T., Tamás, P., Illés, B., Stankevičiūtè, Ž., \& Bányai, Á. (2019). Optimization of municipal waste collection routing: Impact of industry 4.0 technologies on environmental awareness and sustainability. International Journal of Environmental Research and Public Health, 16(4), 634. https://doi.org/10.3390/ijerph16040634

Bhagawati, M. T., Manavalan, E., Jayakrishna, K., \& Venkumar, P. (2019). Identifying Key Success Factors of Sustainability in Supply Chain Management for Industry 4.0 Using DEMATEL Method. In Proceedings of International Conference on Intelligent Manufacturing and Automation (pp. 583-591). Springer, Singapore. https://doi.org/10.1007/978-981-13-24901_54

Bogers, M., Zobel, A. K., Afuah, A., Almirall, E., Brunswicker, S., Dahlander, L., ... \& Hagedoorn, J. (2017). The open innovation research landscape: Established perspectives and emerging themes across different levels of analysis. Industry and Innovation, 24(1), 8-40. https://doi.org/10.1080/13662716.2016.1240068

Bonilla, S., Silva, H., Terra da Silva, M., Franco Gonçalves, R., \& Sacomano, J. (2018). Industry 4.0 and sustainability implications: A scenario-based analysis of the impacts and challenges. Sustainability, 10(10), 3740. https://doi.org/10.3390/su10103740

Buer, S. V., Strandhagen, J. O., \& Chan, F. T. (2018). The link between Industry 4.0 and lean manufacturing: mapping current research and establishing a research agenda. International Journal of Production Research, 56(8), 2924-2940. https://doi.org/10.1080/00207543.2018.1442945

Cakmakci, M. (2019). Interaction in Project Management Approach Within Industry 4.0. In Advances in Manufacturing II (pp. 176-189). Springer, Cham. https://doi.org/10.1007/978-3-030-187156_15

Cezarino, L. O., Liboni, L. B., Oliveira Stefanelli, N., Oliveira, B. G., \& Stocco, L. C. (2019). Diving into emerging economies bottleneck: Industry 4.0 and implications for circular economy. Management Decision. 57, 1-22. https://doi.org/10.1108/MD-10-2018-1084

Cooke-Davies, T. (2002). The "real" success factors on projects. International Journal of Project Management, 20(3), 185-190. https://doi.org/10.1016/S0263-7863(01)00067-9

Dalenogare, L. S., Benitez, G. B., Ayala, N. F., \& Frank, A. G. (2018). The expected contribution of Industry 4.0 technologies for industrial performance. International Journal of Production Economics, 204, 383-394. https://doi.org/10.1016/j.ijpe.2018.08.019 
De Carolis, A., Macchi, M., Negri, E., \& Terzi, S. (2017b, September). A maturity model for assessing the digital readiness of manufacturing companies. In IFIP International Conference on Advances in Production Management Systems (pp. 13-20). Springer, Cham. https://doi.org/10.1007/978-3319-66923-6_2

de FSM Russo, R., \& Camanho, R. (2015). Criteria in AHP: a systematic review of literature. Procedia Computer Science, 55, 1123-1132. https://doi.org/10.1016/j.procs.2015.07.081

de Sousa Jabbour, A. B. L., Jabbour, C. J. C., Foropon, C., \& Godinho Filho, M. (2018). When titans meet-Can industry 4.0 revolutionise the environmentally-sustainable manufacturing wave? The role of critical success factors. Technological Forecasting and Social Change, 132, 18-25. https://doi.org/10.1016/j.techfore.2018.01.017

Durana, P., Kral, P., Stehel, V., Lazaroiu, G., \& Sroka, W. (2019). Quality Culture of Manufacturing Enterprises: A possible way to adaptation to Industry 4.0. Social Sciences, 8(4), 124. https://doi.org/10.3390/socsci8040124

Fengque, P. E. I., Yifei, T. O. N. G., Fei, H. E., \& Dongbo, L. I. (2017). Research on design of the smart factory for forging enterprise in the industry 4.0 environment. Mechanics, 23(1), 146-152. https://doi.org/10.5755/j01.mech.23.1.13662

Fettermann, D. C., Cavalcante, C. G. S., Almeida, T. D. D., \& Tortorella, G. L. (2018). How does Industry 4.0 contribute to operations management?. Journal of Industrial and Production Engineering, 35(4), 255-268. https://doi.org/10.1080/21681015.2018.1462863

Frank, A. G., Dalenogare, L. S., \& Ayala, N. F. (2019). Industry 4.0 technologies: Implementation patterns in manufacturing companies. International Journal of Production Economics, 210, 1526. https://doi.org/10.1016/j.ijpe.2019.01.004

Ghobakhloo, M. (2018). The future of manufacturing industry: a strategic roadmap toward Industry 4.0. Journal of Manufacturing Technology Management, 29(6), 910-936. https://doi.org/10.1108/JMTM-02-2018-0057

Ghobakhloo, M. (2019). Determinants of information and digital technology implementation for smart manufacturing. International Journal of Production Research, 1-22.

https://doi.org/10.1080/00207543.2019.1630775

Haseeb, M., Hussain, H. I., Ślusarczyk, B., \& Jermsittiparsert, K. (2019). Industry 4.0: A solution towards technology challenges of sustainable business performance. Social Sciences, 8(5), 154. https://doi.org/10.3390/socsci8050154

Huang, C. J., Chicoma, T., Denisse, E., \& Huang, Y. H. (2019). Evaluating the Factors that are Affecting the Implementation of Industry 4.0 Technologies in Manufacturing MSMEs, the Case of Peru. Processes, 7(3), 161. https://doi.org/10.3390/pr7030161

Ji, X. Y., Ye, H., Zhou, J. X., \& Deng, W. L. (2016). Digital management technology and its application to investment casting enterprises. China Foundry, 13(5), 301-309.

https://doi.org/10.1007/s41230-016-6011-0 
Kamble, S. S., Gunasekaran, A., \& Gawankar, S. A. (2018). Sustainable Industry 4.0 framework: A systematic literature review identifying the current trends and future perspectives. Process Safety and Environmental Protection, 117, 408-425. https://doi.org/10.1016/j.psep.2018.05.009

Kamble, S. S., Gunasekaran, A., \& Sharma, R. (2018). Analysis of the driving and dependence power of barriers to adopt industry 4.0 in Indian manufacturing industry. Computers in Industry, 101, 107-119. https://doi.org/10.1016/j.compind.2018.06.004

Kamble, S., Gunasekaran, A., \& Dhone, N. C. (2019). Industry 4.0 and lean manufacturing practices for sustainable organisational performance in Indian manufacturing companies. International Journal of Production Research, 1-19. https://doi.org/10.1080/00207543.2019.1630772

Kirchberger, M. A., \& Pohl, L. (2016). Technology commercialization: a literature review of success factors and antecedents across different contexts. The Journal of Technology Transfer, 41(5), 1077-1112. https://doi.org/10.1007/s10961-016-9486-3

Ključnikov, A., Mura, L., \& Sklenár, D. (2019). Information security management in SMEs: Factors of success. Entrepreneurship and Sustainability Issues, 6 (4). http://doi.org/10.9770/jesi.2019.6.4(37)

Leidecker, J. K., \& Bruno, A. V. (1984). Identifying and using critical success factors. Long Range Planning, 17(1), 23-32. https://doi.org/10.1016/0024-6301(84)90163-8

Li, T., Liu, Y., Gao, L., \& Liu, A. (2017). A cooperative-based model for smart-sensing tasks in fog computing. IEEE Access, 5, 21296-21311. https://doi.org/10.1109/ACCESS.2017.2756826

Liu, N., Chow, P. S., \& Zhao, H. (2019). Challenges and critical successful factors for apparel mass customization operations: recent development and case study. Annals of Operations Research, 1-33. https://doi.org/10.1007/s10479-019-03149-7

Lu, S., Xu, C., Zhong, R. Y., \& Wang, L. (2017). A RFID-enabled positioning system in automated guided vehicle for smart factories. Journal of Manufacturing Systems, 44, 179-190.

https://doi.org/10.1016/j.jmsy.2017.03.009

Lucianetti, L., Jabbour, C. J. C., Gunasekaran, A., \& Latan, H. (2018). Contingency factors and complementary effects of adopting advanced manufacturing tools and managerial practices: Effects on organizational measurement systems and firms' performance. International Journal of Production Economics, 200, 318-328. https://doi.org/10.1016/j.ijpe.2018.04.005

Luthra, S., \& Mangla, S. K. (2018). Evaluating challenges to Industry 4.0 initiatives for supply chain sustainability in emerging economies. Process Safety and Environmental Protection, 117, 168179. https://doi.org/10.1016/j.psep.2018.04.018

Maasz, G. J., \& Darwish, H. (2018). Towards an initiative-based industry 4.0 maturity improvement process: master drilling as a case study. South African Journal of Industrial Engineering, 29(3), 92-107. https://doi.org/10.7166/29-3-2052

Manavalan, E., \& Jayakrishna, K. (2019). A review of Internet of Things (IoT) embedded sustainable supply chain for industry 4.0 requirements. Computers e Industrial Engineering, 127, 925-953. https://doi.org/10.1016/j.cie.2018.11.030 
Marnewick, C. \& Marnewick, A. L. (2019). The Demands of Industry 4.0 on Project Teams. IEEE Transactions on Engineering Management. https://doi.org/10.1109/TEM.2019.2899350

Masood, T., \& Egger, J. (2019). Augmented reality in support of Industry 4.0-Implementation challenges and success factors. Robotics and Computer-Integrated Manufacturing, 58, 181195. https://doi.org/10.1016/j.rcim.2019.02.003

Matt, C., Hess, T., \& Benlian, A. (2015). Digital transformation strategies. Business \& information systems engineering, 57(5), 339-343. https://doi.org/10.1007/s12599-015-0401-5

Moeuf, A., Pellerin, R., Lamouri, S., Tamayo-Giraldo, S. e Barbaray, R. (2018). The industrial management of SMEs in the era of Industry 4.0. International Journal of Production Research, 56(3), 1118-1136. https://doi.org/10.1080/00207543.2017.1372647

Moktadir, M. A., Ali, S. M., Kusi-Sarpong, S., \& Shaikh, M. A. A. (2018). Assessing challenges for implementing Industry 4.0: Implications for process safety and environmental protection. Process Safety and Environmental Protection, 117, 730-741. https://doi.org/10.1016/j.psep.2018.04.020

Nagy, J., Oláh, J., Erdei, E., Máté, D., \& Popp, J. (2018). The role and impact of Industry 4.0 and the internet of things on the business strategy of the value chain - the case of Hungary. Sustainability, 10(10), 3491. https://doi.org/10.3390/su10103491

Nicoletti, L., \& Padovano, A. (2019). Human factors in occupational health and safety 4.0: a crosssectional correlation study of workload, stress and outcomes of an industrial emergency response. International Journal of Simulation and Process Modelling, 14(2), 178-195. https://www.inderscienceonline.com/doi/pdf/10.1504/IJSPM.2019.099912

Osei-Kyei R. \& Chan A. P.C. (2015). Review of studies on the Critical Success Factors for Public-Private Partnership (PPP) projects from 1990 to 2013. International Journal of Project Management 33, 1335-1346. https://doi.org/10.1016/j.ijproman.2015.02.008

Piccarozzi, M., Aquilani, B., \& Gatti, C. (2018). Industry 4.0 in management studies: A systematic literature review. Sustainability, 10(10), 3821. https://doi.org/10.3390/su10103821

Pollock, A., \& Berge, E. (2018). How to do a systematic review. International Journal of Stroke, 13(2), 138-156. https://doi.org/10.1177/1747493017743796

RADUJKOVIĆ, Mladen; SJEKAVICA, Mariela. Project management success factors. Procedia engineering, v. 196, p. 607-615, 2017. https://doi.org/10.1016/j.proeng.2017.08.048

Rajput, S., \& Singh, S. P. (2019). Connecting circular economy and Industry 4.0. International Journal of Information Management, 49, 98-113. https://doi.org/10.1016/j.ijinfomgt.2019.03.002

Rauch, E., Dallasega, P., \& Matt, D. T. (2017). Distributed manufacturing network models of smart and agile mini-factories. International Journal of Agile Systems and Management, 10(3-4), 185205. file:///C:/Users/silvi/Downloads/IJASM1003-0401RAUCH_164691.pdf

Rossini, M., Costa, F., Tortorella, G. L., \& Portioli-Staudacher, A. (2019). The interrelation between Industry 4.0 and lean production: an empirical study on European manufacturers. The International Journal of Advanced Manufacturing Technology, 102(9-12), 3963-3976. https://doi.org/10.1007/s00170-019-03441-7 
Scuotto, V., Del Giudice, M., Della Peruta, M. R., \& Tarba, S. (2017). The performance implications of leveraging internal innovation through social media networks: an empirical verification of the smart fashion industry. Technological Forecasting and Social Change, 120, 184-194. https://doi.org/10.1016/j.techfore.2017.03.021

Scuotto, V., Del Giudice, M., Della Peruta, M. R., \& Tarba, S. (2017). The performance implications of leveraging internal innovation through social media networks: an empirical verification of the smart fashion industry. Technological Forecasting and Social Change, 120, 184-194. https://doi.org/10.1016/j.techfore.2017.03.021

Sevinç, A., Gür, Ş., \& Eren, T. (2018). Analysis of the Difficulties of SMEs in Industry 4.0 Applications by Analytical Hierarchy Process and Analytical Network Process. Processes, 6(12), 264. https://doi.org/10.3390/pr6120264

Shinohara, A. C., da Silva, E. H. D. R., de Lima, E. P., Deschamps, F., \& da Costa, S. E. G. (2017). Critical success factors for digital manufacturing implementation in the context of Industry 4.0. In IIE Annual Conference. Proceedings. Institute of Industrial and Systems Engineers (IISE), (pp. 199204). https://www.proquest.com/scholarly-journals/critical-success-factors-digitalmanufacturing/docview/1951123581/se-2 ?accountid=44866

Singh, S., Misra, S. C., \& Chan, F. T. (2019). Establishment of critical success factors for implementation of product lifecycle management systems. International Journal of Production Research, 1-20. https://doi.org/10.1080/00207543.2019.1605227

Slusarczyk, B. (2018) Industry 4.0 - Are you ready? Polish Journal of Management Studies, 17(1), 232248. https://doi.org/10.17512/pjms.2018.17.1.19

Slusarczyk, B., Haseeb, M., \& Hussain, H. I. (2019). Fourth industrial revolution: a way forward to attain better performance in the textile industry. Engineering Management in Production and Services, 11(2), 52-69. https://doi.org/10.2478/emj-2019-0011

Stachová, K., Papula, J., Stacho, Z., \& Kohnová, L. (2019). External partnerships in employee education and development as the key to facing industry 4.0 challenges. Sustainability, 11(2), 345. https://doi.org/10.3390/su11020345

Tao, F., \& Zhang, M. (2017). Digital twin shop-floor: a new shop-floor paradigm towards smart manufacturing. IEEE Access, 5, 20418-20427. https://doi.org/10.1109/ACCESS.2017.2756069

Theorin, A. , Bengtsson, K., Provost, J., Lieder, M., Johnsson, C., Lundholm, T., \& Lennartson, B. (2017). An event-driven manufacturing information system architecture for Industry 4.0. International Journal of Production Research, 55(5), 1297-1311. https://doi.org/10.1080/00207543.2016.1201604

Tsai, W. H. (2018). Green production planning and control for the textile industry by using mathematical programming and industry 4.0 techniques. Energies, 11(8), 2072. https://doi.org/10.3390/en11082072

Tsai, W. H., \& Lu, Y. H. (2018). A Framework of production planning and control with carbon tax under industry 4.0. Sustainability, 10(9), 3221. https://doi.org/10.3390/su10093221 
Türkeș, M. C., Oncioiu, I., Aslam, H. D., Marin-Pantelescu, A., Topor, D. I., \& Căpușneanu, S. (2019). Drivers and Barriers in Using Industry 4.0: A Perspective of SMEs in Romania. Processes, 7(3), 153. https://doi.org/10.3390/pr7030153

Varela, L., Araújo, A., Ávila, P., Castro, H., \& Putnik, G. (2019). Evaluation of the Relation between Lean Manufacturing, Industry 4.0, and Sustainability. Sustainability, 11(5), 1439.

https://doi.org/10.3390/su11051439

Xu, L. D., Xu, E. L., \& Li, L. (2018). Industry 4.0: state of the art and future trends. International Journal of Production Research, 56(8), 2941-2962. https://doi.org/10.1080/00207543.2018.1444806

Zhang, Y., Qian, C., Lv, J., \& Liu, Y. (2016). Agent and cyber-physical system based self-organizing and self-adaptive intelligent shopfloor. IEEE Transactions on Industrial Informatics, 13(2), 737-747. https://doi.org/10.1109/TII.2016.2618892 